\title{
INVENTARIO DE REACTIVIDAD INTERPERSONAL. ADAPTACIÓN CULTURAL Y ANÁLISIS PSICOMÉTRICO EN UNA MUESTRA DE UNIVERSITARIOS EN COLOMBIA
}

\author{
Reynel Alexander Chaparro Clavijo ${ }^{a 1}{ }^{\mathbb{D}}, \&$ Carlos Alejandro \\ Pineda-Roa ${ }^{b}$ (i) ${ }^{2}$
}

Universidad Nacional de Colombia, Bogotá, Colombia. ${ }^{\text {a }}$ Universidad del Magdalena, Santa Marta, Colombia. ${ }^{\mathrm{b}}$

\begin{abstract}
RESUMEN
Se realizó la validación cultural del Inventario de Reactividad Interpersonal (IRI), traduciendo al español y revisando las propiedades psicométricas en sus modelos de dos factores, cuatro factores y cuatro factores + un factor de segundo orden. A través de muestreo no probabilístico participaron 386 estudiantes universitarios (edades entre 18 y 30 años, $\mathrm{M}=20.2$; $\mathrm{DE}=2.6$ ). La confiabilidad de las dimensiones resultó adecuada (Alfa de Cronbach $0.66<\alpha<0.78$; Omega de McDonald $0.70<\Omega<0.80$ ). El Análisis Factorial Confirmatorio mostró indicadores con buen ajuste al modelo de cuatro factores y cuatro factores más un factor de segundo orden e insuficientes en el modelo de dos factores. Las mujeres mostraron mayores puntajes en las dimensiones afectivas, mientras que los hombres mostraron mayores puntajes en las dimensiones cognitivas. La versión del IRI reducida a 22 ítems conserva buenas propiedades psicométricas en la población joven colombiana.
\end{abstract}

\section{Palabras Claves}

empatía disposicional; validación cultural; Escala de Reactividad Interpersonal

\begin{abstract}
A cultural validation of the Interpersonal Reactivity Inventory (IRI) was carried out, translating into Spanish and reviewing its psychometrical properties, evaluating two-factor, four-factor and four-factor + one general-factor models. 386 university students participated in a non-probabilistic sampling (18 - 30 years old, $\mathrm{M}=20.2 ; \mathrm{SD}=2.6$ ). The reliability of the dimensions was adequate (Cronbach's alpha $0.66<\alpha$ $<0.78$; McDonald's Omega $0.70<\Omega<0.80$ ). Confirmatory Factor Analysis showed good fit to four-factor model and for four-factor + one general-factor model and insufficient in two-factor model. Women showed higher scores in affective dimensions whereas men showed higher scores in cognitive dimensions. IRI 22 items reduced version shows good psychometric properties in young Colombian population.
\end{abstract}

Keywords

dispositional empathy; cultural validation; Interpersonal Reactivity Index

\footnotetext{
${ }^{1}$ Correspondence about this article should be addressed to Reynel Alexander Chaparro Clavijo: rachaparroc@unal.edu.co

${ }^{2}$ Agradecimientos a Colciencias por financiación de Doctorado en Psicología. Reynel Alexander Chaparro-Clavijo convocatoria 647 de 2014; Carlos Alejandro Pineda Roa convocatoria 727 de 2015.
} 


\section{INTERPERSONAL REACTIVITY INDEX: CULTURAL ADAPTATION AND PSYCHOMETRIC ANALYSIS IN UNDERGRADUATE COLOMBIAN STUDENTS}

\section{Introducción}

Si bien hay un acuerdo general sobre la definición de la empatía como ponerse uno mismo "en los zapatos de otros", esta es considerada como un conjunto de constructos relacionados que se refieren a la responsividad hacia otros (Davis, 1983). En esta forma de responder hacia las demás personas, cobra relevancia la posibilidad de describir la capacidad de compartir y entender sus pensamientos y sentimientos, así como las conductas relativas a la empatía (Auné, Facundo, \& Attorresi, 2017). La importancia del estudio de la empatía es relevante en tanto es un componente base para la interacción social, pues se relaciona con la conducta prosocial (Auné, Abal, \& Attorresi, 2016), por lo que se infiere un grado de disposición empática desarrollado a lo largo del tiempo (Duan, \& Hill, 1996; Davis, 1980).

La empatía disposicional se ha separado en dos grandes énfasis comprensivos, que incluyen un componente cognitivo en el que hay un acercamiento a estados mentales, aspectos imaginativos o de comprensión del otro sin que se experimenten los sentimientos de esa persona (Hogan, 1969) y, por otra parte, un componente afectivo o emocional, en el que se destaca la receptividad a la experiencia emocional de otra persona (Mehrabian, \& Epstein, 1972).

Algunas propuestas de evaluación de la empatía disposicional han enfatizado en esta separación, evaluando solamente el componente cognitivo como lo hace el Hogan Empathy Scale (Hogan, 1969) o el componente afectivo, por ejemplo, a través del Questionnaire Measure of Emotional Empathy (Mehrabian, \& Epstein, 1972) o el Balanced Emotional Empathy Scale (Mehrabian, 1997). Sin embargo la empatía disposicional, entendida como un constructo multidimensional que integra dimensiones de lo cognitivo y lo afectivo, ha sido ampliamente estudiada en la subdivisión planteada por Davis (1980) a través del Interpersonal Reactivity Index - el Inventario de Reactividad Interpersonal (IRI).

El IRI es una de las de las medidas de autoinforme más utilizadas para evaluar la empatía disposicional y con más soporte investigativo de su utilidad en el campo del estudio de la empatía (Mestre, Frías, \& Samper, 2004; Fernández-Pinto, López-Pérez, \& Márquez, 2008), pues integra el componente cognitivo con las subescalas fantasía y toma 
de perspectiva, y el componente afectivo con las subescalas preocupación empática y aflicción personal. Estos componentes aunque diferentes, están relacionados entre sí.

La Fantasía refiere a la tendencia a identificarse con personajes de ficción, por lo que indica una capacidad imaginativa de la persona para ponerse en situaciones ficticias. La Toma de Perspectiva es la habilidad para comprender el punto de vista de otras personas. La Preocupación Empática es la tendencia a experimentar sentimientos de compasión y preocupación por otra persona (se trata de sentimientos "orientados al otro"). La Aflicción Personal es la ansiedad que se experimenta al ser testigo de un suceso desagradable para otra persona (se trata de sentimientos "orientados al yo") (Davis, 1980).

Son ampliamente reconocidas las diferencias de género, pues consistentemente se reporta un mayor puntaje en mujeres que en hombres (Davis, 1980; Mestre, Samper, Tur, \& Díez, 2001; Mestre, Samper, \& Frías, 2002; Mestre, et al., 2004), siendo en algunos estudios más altos los puntajes en mujeres en las dimensiones preocupación empática, toma de perspectiva y aflicción personal (Eisenberg, Miller, Shell, Mcnalley, \& Shea, 1991) y en otros en fantasía, preocupación empática y aflicción personal (Retuerto, 2004).

Las diferentes formas de comprender la empatía disposicional con el IRI abarcan un modelo global de empatía (Martin, Flasbeck, Brown, \& Brüne, 2017; Aridag, \& Yuksel, 2010; Wang, Zhang, Dong, Chen, Zheng et al., 2014; Airagnes, Consoli, De Morlhon, Galliot, Lemonge et al., 2014; Molina, Arikawa, \& Templer, 2013; Bilgic, Bilgiç, Hergüner, \& Altınyazar, 2015) un modelo de dos factores (cognitivo y afectivo) que utiliza principalmente las dos subescalas, preocupación empática y toma de perspectiva (Mohamed Azahar, Mohd Fakri, \& Mat Pa, 2014; Tamura, Sugiura, Sugiura, \& Moriya, 2016; Cox, Uddin, Di Martino, Castellanos, Milham et al., 2012), así como un modelo de cuatro factores diferenciados (cada subescala del IRI) y un factor de segundo orden (Montag, Brockmann, Lehmann, Müller, Rujescu et al., 2012; Song, \& Shi, 2017; Stratton, Saunders, \& Elam, 2008).

El modelo de dos factores ha recibido amplio soporte teórico, al plantearse como una forma de análisis de la multidimensionalidad de la empatía. No obstante, estudios recientes que buscan su validez psicométrica desmienten este modelo dual (Chrysikou, \& Thompson, 2016) y se aboga por una reevaluación de los análisis que se hacen implementando el IRI para delimitar la empatía cognitiva y afectiva. Otros análisis han confirmado la estructura de los cuatro factores propuestos por Davis (Pérez, de Paul, Etxeberría, Paz, \& Torres, 2003), así como el de cuatro factores y un factor de segundo orden (Cliffordson, 2002; Pulos, Elison, \& Lennon, 2004). 
Por otra parte se contemplan versiones reducidas a 26 ítems (Sampaio, Guimarães, Camino, Formiga, \& Menezes, 2011), 24 ítems (Limpo, Alves, \& Catro, 2010; Chiang, Hua, Tam, Chao, \& Shiah, 2014), 16 ítems (Ingoglia, Lo Coco, \& Albiero, 2016) y 15 ítems (Braun, et al., 2015), que pretenden una depuración de ítems al mismo tiempo que la búsqueda de una mayor robustez de la prueba y economía en su aplicación.

Sin embargo, es preocupante en el proceso de adaptación cultural la alteración de la prueba sin la debida justificación psicométrica (Koller, Camino, \& Ribeiro, 2001) y la utilización de la traducción de una sola vía, sin tener en cuenta una apropiada validación cultural (Samper, Díez, \& Martí, 1998; Carrasco, Delgado, Barbero, Holgado, \& del Barrio, 2011; Artacho, López, Molina, Ortiz, Rosado et al., 2012; Díaz, Rodríguez, \& Santa Cruz, 2013; Braun, Rosseel, Kempenaers, Loas, \& Linkowski, 2015; Kostić, Nešić, Stanković, Žikić, \& Marković, 2016).

A la luz de los antecedentes planteados y teniendo en cuenta que (hasta donde se conoce) no hay investigaciones relacionadas en el contexto colombiano que aprovechen el potencial del estudio y comprensión de la empatía desde el IRI, el siguiente trabajo busca realizar 1) una adecuada validación cultural del IRI en el contexto colombiano; 2) examinar la estructura factorial del IRI en sus modelos de dos factores, cuatro factores y cuatro factores + un factor de segundo orden; y 3) explorar la posibilidad de una versión reducida del IRI. Los resultados de este estudio buscan aportar a la comprensión psicométrica del IRI en el contexto colombiano, revisar la estabilidad de los modelos evaluados, así como motivar las investigaciones locales que tienen en cuenta el concepto de empatía disposicional con una propuesta de una versión reducida del IRI.

\section{Método}

\section{Diseño}

El presente estudio es de tipo instrumental de naturaleza psicométrica (Montero, \& León, 2007).

\section{Participantes}

La aplicación del IRI se realizó en estudiantes universitarios de pregrado de tres universidades de Bogotá, una pública y dos privadas, procedentes de diversos lugares del país y residentes en Bogotá y sus alrededores. El muestreo fue no probabilístico por 
disponibilidad y en su recolección colaboraron estudiantes de psicología previamente entrenados en el diligenciamiento de la prueba.

Se obtuvo la participación voluntaria de 386 estudiantes, 216 mujeres (56\%) y 170 hombres (44\%). La edad promedio de la muestra total osciló entre 18 y 30 años (Media $=20.2, \mathrm{DE}=2.6)$. La mayoría de estudiantes eran solteros $(95 \%)$ y pertenecían a los estratos dos $(37.1 \%)$, tres $(48.3 \%)$ y cuatro $(9.8 \%)$. Sólo el $1,6 \%$ era de estrato cinco y seis y $3.2 \%$ pertenecía a estrato uno ${ }^{3}$.

\section{Instrumentos}

Se aplicó inicialmente una ficha socio-demográfica en la que se indagaba sobre la edad, el género, el estrato social, el nivel educativo y el estado civil. Posteriormente se aplicó la versión en español del IRI. El instrumento tiene un total de 28 ítems con opciones de respuesta del 1 al 5, donde 1 es No me describe muy bien y 5 es Me describe muy bien. Los ítems de la prueba se presentaron a lápiz y papel. En las instrucciones se les invitó a leer cada una de las afirmaciones, las cuales preguntan por sus pensamientos y sentimientos en una variedad de situaciones, y posteriormente generar la respectiva puntuación.

\section{Procedimiento de traducción y validación cultural del IRI}

Se realizó una revisión de dos traducciones hechas al español (de España) por Pérez et al., (2003) y Mestre et al., (2004). La revisión fue realizada por tres psicólogas clínicas con estudios posgraduales en el área y con más de diez años de experiencia, dos psicólogas bilingües de nacionalidad colombiana y una bilingüe con doble nacionalidad (estadounidense y colombiana), quienes además contrastaron y revisaron la versión original en inglés.

En el proceso de revisión de la traducción se aplicaron la escala de calificación de la claridad de los ítems (adaptado de Escobar, \& Cuervo, 2008) y las dimensiones de error en la traducción de pruebas (adaptado de Solano, Contreras, \& Backhoff, 2006).

Se realizó un proceso de reconciliación de la evaluación y del proceso de traducción, resolviendo las discrepancias y buscando el acuerdo entre hábitos, discursos

\footnotetext{
${ }^{3}$ En Colombia la estratificación socioeconómica es una forma de determinar la capacidad económica y las características sociales de un grupo poblacional según el lugar de vivienda. El rango es de 1 a 6 , agrupando en el rango superior la mayor capacidad económica.
} 
y preferencias individuales, para tener una versión conciliada en esta primera traducción de todos los ítems.

Así también, para asegurar la equivalencia conceptual en la traducción, se hizo el proceso de retraducción de la versión traducida al español (de Colombia) con el apoyo de un profesional certificado en traducción español-inglés de nacionalidad estadounidense. Posteriormente se contó con la ayuda y el aval del autor de la prueba, Mark Davis, para realizar la revisión final de la retraducción de la versión colombiana y su uso posterior en la investigación. Se prestó especial atención a las diferencias entre las versiones lingüísticas, para asegurar la correspondencia de cada ítem traducido de nuevo a la versión original. En este proceso se hicieron ajustes a la traducción de los ítems 19 (diferencias entre la comprensión y las palabras effective y efficient) y 22 (soft-hearted y sensitive).

Posteriormente, esta versión del IRI traducida al español se sometió a una prueba piloto de 25 estudiantes universitarios de diferentes carreras, con el propósito de evaluar la redacción, comprensión y tiempo de respuesta. Se realizó asimismo una evaluación grupal para evaluar e identificar cualquier problema, poniendo especial énfasis en la comprensión idiomática y conceptual. De este proceso no se tuvieron aspectos a corregir (ver versión definitiva en el anexo A).

\section{Análisis de datos}

Se capturó la información en Excel y se pasó a SPSS versión 22. Se reportan los resultados de confiabilidad por consistencia interna a través del Omega de McDonald y el Alfa de Cronbach, y de dimensionalidad del instrumento mediante el Análisis Factorial Confirmatorio (AFC), previa extracción del número de factores a retener según el Análisis paralelo (AP) de Horn (1965). El Omega de McDonald y el AP fueron calculados a través del software libre Factor versión 10.3.01 (Lorenzo-Seva, \& Ferrando, 2015). El AFC fue realizado a través de Lisrel versión 8.8 (Jöreskog, Karl, \& Sörbom, 2009).

Para conocer la bondad del ajuste del AFC se calcularon la prueba de chi cuadrado de Satorra-Bentler $\chi^{2}$ S-B, con grados de libertad (gl) y valor de probabilidad (p), y los coeficientes RMSEA (Root Mean Square Error of Approximation, raíz del cuadrado media del error de aproximación) e intervalo de confianza del 90\% (IC90\%), CFI (Comparative Fit Index, índice comparativo de ajuste), AGFI (Adjusted Goodness of Fit Index, índice de bondad de ajuste ajustado) TLI o NNFI (Tucker-Lewis index) y SRMS 
(Standardized Mean Square Residual, residuo cuadrado promedio estandarizado). Para chi cuadrado se espera que la razón $\chi^{2} /$ gl sea inferior a 3; para RMSEA y SRMR $<0,06$, para GFI, AGFI, CFI y TLI valores $>0,89$.

\section{Aspectos éticos}

Se tuvo en cuenta lo dispuesto en el Código Deontológico de la Psicología en Colombia (Ley 1090 de 2006) y lo dispuesto en la Resolución 8430 de 1993 para el Desarrollo de Investigación en Ciencias de la Salud, que señala que la presente investigación se caracterizó como sin riesgo. Se aplicó el consentimiento informado en la prueba piloto y previo a la recolección de los datos, en el que se garantizó la protección de la confidencialidad y la libre participación (pudiendo salir del proceso en cualquier momento y sin ningún tipo de repercusión), respetando el derecho de los participantes de conocer los resultados, de autorizar la publicación de los hallazgos y demás interpretaciones hechas con los datos con base a las conclusiones y recomendaciones del proceso de validación. En este proceso de recopilación de información no se ofreció remuneración alguna a los participantes.

\section{Resultados}

La tabla 1 muestra los estadísticos descriptivos de las sub-escalas. Se presentan los resultados del AP de Horn, cargas factoriales de la versión mejorada con la confiabilidad total y por subescala, luego los hallazgos de estructura interna de la prueba mediante AFC y por último los análisis de validez discriminante según la variable sexo. Tabla 1.

Estadísticos descriptivos de las subescalas y la prueba global IRI

\begin{tabular}{ccccc}
\hline Subescalas & Media & DT & Min & Max \\
\hline Fantasía & 14.29 & 4.23 & 5 & 25 \\
Toma de perspectiva & 17.18 & 3.54 & 5 & 25 \\
Preocupación empática & 25 & 4.82 & 10 & 35 \\
Aflicción personal & 16.52 & 4.18 & 6 & 27 \\
Empatía & 73 & 10.6 & 45 & 103 \\
\hline
\end{tabular}

Fuente: Elaboración propia.

\section{Análisis de los resultados del AP de Horn}

El AP nos permite realizar una selección de componentes que no dependa de la longitud del test o del tamaño muestral. Además, posibilita una confirmación del número 
de componentes a retener (Horn, 1965). El AP ha ganado un importante terreno en la psicometría como una mejor alternativa a la regla de Kaiser, que establece retener un número de componentes o factores en un Análisis Factorial Exploratorio cuyos autovalores sean mayores que 1 . La regla de Kaiser ha sido bastante criticada, ya que tiende a subestimar o sobreestimar el número de factores o componentes. El procedimiento establece aceptar aquellos factores o componentes, siempre que se cumpla que el valor propio de los datos reales supere el valor propio aleatorio o simulado (Brett, Brown, \& Onsman, 2010). La tabla 2 muestra los resultados de AP para el IRI y la proporción de varianza explicada por cada factor aceptado, así como la proporción de varianza acumulada explicada por los cuatro factores retenidos.

Tabla 2.

Resultados del Análisis Paralelo de Horn de los ítems de IRI

\begin{tabular}{llllll}
\hline Factor & Valor propio & $\begin{array}{l}\text { Valor propio } \\
\text { percentilar }\end{array}$ & Decisión & $\begin{array}{l}\text { Proporción } \\
\text { Varianza } \\
\text { explicada }\end{array}$ & $\begin{array}{l}\text { Proporción } \\
\text { varianza } \\
\text { acumulada }\end{array}$ \\
\hline 1 & $5.36942^{*}$ & 1.58855 & Aceptar & .22454 & .489 \\
2 & $3.06114^{*}$ & 1.50041 & Aceptar & .1096 & \\
3 & $2.24259^{*}$ & 1.43891 & Aceptar & .08885 & \\
4 & $1.58008^{*}$ & 1.38194 & Aceptar & .06657 & \\
5 & 1.20398 & 1.33352 & Rechazar & & \\
6 & 1.03705 & 1.29219 & Rechazar & & \\
7 & 1.01192 & 1.24896 & Rechazar & & \\
\hline
\end{tabular}

Fuente: Elaboración propia. *Los resultados del AP sugieren retener cuatro factores.

\section{Análisis de los resultados sobre confiabilidad}

Los resultados muestran una confiabilidad adecuada, medida por el Alfa de Cronbach y el Omega de McDonald para el constructo general (empatía) y sus subescalas, lo que revela una alta intercorrelación de los ítems, indicando que cada subescala da cuenta de un constructo psicológicamente interpretable y que estas engloban de modo consistente lo que se puede denominar como la empatía disposicional (Tabla 3). Además, seis ítems fueron excluidos por arrojar valores de correlación ítem total corregida inferior a 0.30. (Ver en el anexo A los ítems retenidos y los excluidos). Como se aprecia en la tabla 3, la dimensión con mejor confiabilidad es preocupación empática, la cual conservó todos los ítems de la versión original. Adicionalmente, las cargas factoriales de la versión mejorada de 22 ítems oscilaron entre 0.42 y 0.88 para todos los factores, con excepción del ítem 7 que tuvo una carga factorial de 0.36 en el factor fantasía. Recuérdese que entre más cercanas sean estas a 1, más importante es el ítem para el factor. 
Tabla 3.

Cargas factoriales $\lambda$ y confiabilidad por factor de la nueva versión propuesta del IRI

\begin{tabular}{|c|c|c|c|c|}
\hline \multirow[t]{2}{*}{ Ítems } & \multicolumn{4}{|c|}{ Factores } \\
\hline & $\begin{array}{c}\text { Toma de } \\
\text { perspectiva }\end{array}$ & Fantasía & $\begin{array}{l}\text { Preocupación } \\
\text { empática }\end{array}$ & $\begin{array}{l}\text { Aflicción } \\
\text { personal }\end{array}$ \\
\hline Ítem 8 & 0,45 & & & \\
\hline Ítem 11 & 0,64 & & & \\
\hline Ítem 21 & 0,58 & & & \\
\hline Ítem 25 & 0,60 & & & \\
\hline Ítem 28 & 0,58 & & & \\
\hline Ítem 5 & & 0,63 & & \\
\hline Ítem 7 & & 0,36 & & \\
\hline Ítem 16 & & 0,68 & & \\
\hline Ítem 23 & & 0,88 & & \\
\hline Ítem 26 & & 0,68 & & \\
\hline Ítem 2 & & & 0,71 & \\
\hline Ítem 4 & & & 0,42 & \\
\hline Ítem 9 & & & 0,53 & \\
\hline Ítem 14 & & & 0,57 & \\
\hline Ítem 18 & & & 0,52 & \\
\hline Ítem 20 & & & 0,75 & \\
\hline Ítem 22 & & & 0,63 & \\
\hline Ítem 6 & & & & 0,61 \\
\hline Ítem 10 & & & & 0,59 \\
\hline Ítem 17 & & & & 0,62 \\
\hline Ítem 24 & & & & 0,55 \\
\hline Ítem 27 & & & & 0,63 \\
\hline No. de ítems & 5 & 5 & 7 & 5 \\
\hline $\begin{array}{c}\text { Alfa de Cronbach: } \\
(\mathrm{n}=22 \text { ítems, } \alpha=0.78)\end{array}$ & 0,66 & 0,74 & 0,75 & 0,69 \\
\hline $\begin{array}{l}\text { Omega de McDonald: ( } \mathrm{n}= \\
22 \text { ítems, } \Omega=0.80 \text { ) }\end{array}$ & 0,70 & 0,75 & 0,80 & 0,70 \\
\hline
\end{tabular}

Fuente: Elaboración propia.

\section{Análisis de los resultados sobre validez de constructo o estructura interna}

El AFC arrojó un ajuste aceptable o relativamente bueno en las dos versiones, tanto en el modelo de cuatro factores como el de cuatro factores y uno de segundo orden, siendo ligeramente mejores algunos de los índices del modelo de 4 factores, como se aprecia en las tablas 4 y 5 . 
Tabla 4.

AFC del IRI, versión 28 ítems

\begin{tabular}{cccccccccc}
\hline Modelo & $\mathrm{S}-\mathrm{B} \chi^{2}$ & $\mathrm{gl}$ & NNFI & CFI & GFI & AGFI & SRMR & RMSEA & IC90\%RMSEA \\
\hline Dos factores & 1861.42 & 349 & .70 & .72 & .85 & .82 & .12 & .10 & $(0.099 ; 0.11)$ \\
$\begin{array}{c}\text { Cuatro factores } \\
\text { Cuatro factores y }\end{array}$ & 798.16 & 344 & .91 & .92 & .91 & .89 & .089 & .057 & $(.052 ; .063)$ \\
$\begin{array}{c}\text { un factor de } \\
\text { segundo orden }\end{array}$ & 849.91 & 346 & .90 & .91 & .90 & .88 & .095 & .060 & $(.055 ; .065)$ \\
\hline
\end{tabular}

Fuente: Elaboración propia

Método de estimación: DWLS; gl: grados de libertad.

Todos los $\chi^{2}: \mathrm{P}<0.001$

El índice GFI mide cuánto mejor se ajusta el modelo a los datos comparados con la ausencia de cualquier modelo, así también indica la varianza común entre el modelo propuesto y los datos. Es aceptable si es igual o superior a 0,90, como se aprecia en las tablas 4 y 5, aunque los valores de los índices de ajuste son mejores en la versión mejorada de 22 ítems. Para el caso de RMSEA, se presenta un buen ajuste si es igual o inferior a 0,06 (Hu, \& Bentler, 1999). El ajuste es razonablemente bueno si es igual o inferior a 0,08 (Browne, \& Cudeck, 1993). En efecto, en ambas versiones, los índices RMSEA son buenos. El SRMR representa el valor residual promediado entre la matriz de varianzascovarianzas del modelo propuesto y la matriz de varianzas-covarianzas de la matriz de datos, suele exigirse que sea igual o inferior a 0,08 (Hu, \& Bentler, 1999), por lo que para ambos modelos de la versión mejorada de 22 ítems, este índice está también dentro de los límites exigidos.

Tabla 5.

AFC del IRI, nueva versión de 22 ítems

\begin{tabular}{cccccccccc}
\hline Modelo & S-B $\chi^{2}$ & gl & NNFI & CFI & GFI & AGFI & SRMR & RMSEA & IC90\% \\
\hline Dos factores & 1280.06 & 208 & .72 & .75 & .87 & .85 & .12 & .114 & $(0.11 ; 0.12)$ \\
Cuatro factores & 468.53 & 203 & .93 & .94 & .94 & .92 & .081 & .057 & $(.050 ; .064)$ \\
$\begin{array}{c}\text { Cuatro factores y un solo } \\
\text { factor de segundo orden }\end{array}$ & 507.86 & 205 & 0.92 & .93 & .93 & .91 & .089 & .061 & $(.054 ; .067)$ \\
\hline
\end{tabular}

Fuente: Elaboración propia

Método de estimación: DWLS; gl: grados de libertad.

Todos los $\chi 2: \mathrm{P}<0.001$

Se puede afirmar además que los dos modelos anidados, 4 factores y $4+1$ factores, son equivalentes dada la diferencia del CFI de 0.01 (tabla 5). Es preciso señalar la existencia de un factor de segundo orden, el cual podemos denominar empatía, según los resultados del modelo de 4+1 factores de la versión mejorada. Además, a pesar de que el modelo hipotetiza que dos de las cuatro escalas son de naturaleza cognitiva y dos de naturaleza afectiva, los índices encontrados para este modelo bifactorial no son adecuados. 


\section{Análisis de los resultados sobre validez discriminante}

Los datos de las dimensiones del IRI no se distribuyeron normalmente (K.S $=.05$, $\mathrm{p}<.05)$. En consecuencia, se procedió a calcular las diferencias de rangos de medias para el IRI y sus subescalas mediante la prueba no paramétrica U de Mann Whitney para dos muestras independientes como el caso de la variable sexo. La tabla 6 muestra los resultados por subescala y el total (empatía), en el cual se aprecia una tendencia clara en las mujeres a puntuar más alto en dimensiones afectivas como preocupación empática y aflicción personal, mientras que los hombres muestran puntajes más altos en las dimensiones cognitivas, aunque sólo fue significativa la dimensión fantasía.

Tabla 6.

Resultados de IRI y sus dimensiones según la variable sexo $(n=386,216$ mujeres y 170 hombres)

\begin{tabular}{cccccc}
\hline Dimensión & Sexo & $\begin{array}{c}\text { Rango } \\
\text { promedio }\end{array}$ & $\begin{array}{c}\text { Suma de } \\
\text { rangos }\end{array}$ & $\begin{array}{c}\text { U Mann } \\
\text { Whitney }\end{array}$ & $\mathrm{p}$ \\
\hline Toma de & Mujer & 189.34 & 40898.50 & & \\
perspectiva & Hombre & 198.78 & 33792.50 & 17462.5 & 0.408 \\
Fantasía & Mujer & 184.21 & 39789.50 & & \\
& Hombre & 205.30 & 34901.50 & 16353.5 & 0.065 \\
Preocupación & Mujer & 214.47 & 46324.00 & & \\
empática & Hombre & 166.86 & 28366.50 & 13381 & 0.000 \\
Aflicción & Mujer & 221.20 & 47779.00 & & \\
personal & Hombre & 158.31 & 26912.00 & 12377 & 0.000 \\
Empatía & Mujer & 208.34 & 45001.00 & & \\
& Hombre & 174.64 & 29689.50 & 15154.5 & .003 \\
\hline
\end{tabular}

Fuente: Elaboración propia

\section{Discusión}

Teniendo en cuenta que las diferencias culturales pueden influir en la validez y la confiabilidad de la aplicación de las pruebas desarrolladas en otros países, se realizó la validación cultural del IRI en población joven colombiana, que integra y evalúa las dos versiones al español previamente identificadas (Pérez et al., 2003; Mestre et al., 2004), con un apropiado análisis de la traducción, retraducción y el apoyo del aval del autor de la prueba, superando otros estudios que han omitido este proceso (Fernández, Dufey, \& Kramp, 2011) o en el que sólo se ha hecho una traducción de una vía (Samper, Díez, \& Martí, 1998; Carrasco, Delgado, Barbero, Holgado, \& del Barrio, 2011; Artacho, López, Molina, Ortiz, Rosado et al., 2012; Díaz, Rodríguez, \& Santa Cruz, 2013; Braun, Rosseel, Kempenaers, Loas, \& Linkowski, 2015; Kostić, Nešić, Stanković, Žikić, \& Marković, 2016). 
El IRI muestra una buena consistencia interna utilizando los dos criterios de confiabilidad aplicados (Omega de McDonald y el Alfa de Cronbach). Se sugieren estudios posteriores que evalúen otros procedimientos que incluyan el test re-test y formas paralelas (con su debida adaptación cultural).

El AFC apoya el modelo de cuatro factores propuesto por Davis (1980), así como el de cuatro factores y un factor de segundo orden (Cliffordson, 2002), lo que confirma la conceptualización de la empatía como un fenómeno multidimensional y relacionado. En nuestro caso se considera un mejor ajuste en el modelo de cuatro factores para la muestra analizada. También es de resaltar que debido al pobre ajuste encontrado en el modelo de dos factores, se recomienda no hacer análisis del IRI diferenciando la empatía cognitiva y la afectiva. Estos resultados son consistentes con lo reportado previamente por Chrysikou, \& Thompson (2016).

La propuesta de una versión reducida del IRI a 22 ítems muestra adecuados índices de ajuste a los modelos de cuatro factores y cuatro factores más un factor de segundo orden. En efecto, los índices CFI, GFI, AGFI y NNFI están por encima de 0,90 y SRMR y RMSEA por debajo de 0.08, lo que cumple con lo estipulado por Hu y Bentler (1999) para considerar un ajuste aceptable. Asimismo, la razón $\chi^{2} / \mathrm{gl}$ fue inferior a 3 en ambos modelos. Además, estos dos modelos resultaron equivalentes a pesar de que uno impone más restricciones (4+1 factores) que el otro (4 factores). Al respecto, cuando la diferencia entre los índices CFI resulta menor o igual a 0,01, se dice que los dos modelos son equivalentes (véase Cheung, \& Rensvold, 2002).

Llama la atención el ítem 15: If I'm sure I'm right about something, I don't waste much time listening to other people's arguments - "Si estoy seguro/a que tengo la razón en algo, no pierdo mucho tiempo escuchando los argumentos de los demás" que ha sido recurrentemente descartado en varios estudios (Limpo et al., 2010; Chiang et al., 2014; Braun et al., 2015; Ingoglia et al., 2016), seguido por el ítem 1: I daydream and fantasize, with some regularity, about things that might happen to me - "Con cierta regularidad sueño y fantaseo acerca de las cosas que me podrían suceder", que ha sido recurrentemente descartado en varios estudios (Limpo et al., 2010; Braun et al., 2015; Ingoglia et al., 2016), el ítem 12: Becoming extremely involved in a good book or movie is somewhat rare for me - "Es raro para mí involucrarme completamente en un buen libro o película", el ítem 3: I sometimes find it difficult to see things from the "other guy's" point of view - "A veces encuentro difícil ver las cosas desde el punto de vista de otra persona" (Braun et al., 2015; Ingoglia et al., 2016) y el ítem 19: I am usually pretty 
effective in dealing with emergencies - "Normalmente soy bastante efectivo/a para manejar emergencias" (Chiang et al., 2014; Ingoglia et al., 2016). Estudios posteriores que impliquen la valoración de versiones reducidas del IRI explorarán la pertinencia de continuar incluyendo estos ítems.

Si bien se sigue confirmando que el IRI es sensible a las diferencias de género por la predominancia de mayores puntajes en mujeres como característica fundamental de la muestra analizada, los hombres mostraron mayores puntajes en las dimensiones cognitivas (fantasía y toma de perspectiva). Aunque sólo fue significativo en fantasía, en este estudio se llama la atención sobre el cambio en la interpretación según el género, especialmente en la subescala fantasía. Estudios posteriores deberán confirmar la estabilidad o el cambio en las diferencias por género, teniendo en cuenta el efecto cambiante en los roles de género (por ejemplo en la socialización y creencias de género), los estudios en masculinidades y otras variables que pueden ser contextualmente claves, como por ejemplo el efecto de políticas públicas y educativas en la percepción de las diferencias entre hombres y mujeres.

Se espera que esta versión adaptada del IRI fomente la investigación hispanohablante en empatía en sus múltiples áreas y énfasis de investigación, así como también el mejoramiento del IRI al incluir muestras más extensas y con otras características sociodemográficas que incluyan diferentes rangos de edad, pues consistentemente los estudios del IRI (al igual que este) están centrados en personas jóvenes. 


\section{Referencias}

Airagnes, G., Consoli, S.M., De Morlhon, O., Galliot, A.M., Lemonge, C., \& Jaury, P. (2014). Appropriate training based on Balint groups can improve the empathic abilities of medical students: A preliminary study. Journal of Psychosomatic research, 76, 426-429. http://doi.org/10.1016/j.jpsychores.2014.03.005

Aridag, N.C., \& Yuksel, A. (2010). Analysis of the relationship between moral judgment competences and empathic skills of university students. Educational Sciences: Theory \& Practice, 10(2), 707-724.

Artacho, F., López, M., Molina, A.M., Ortiz, M.V., Rosado, J.I., Ruiz, M.V., \& Sillero, M. (2012). La medida de la empatía en el alumnado de la Facultad de Ciencias de la Educación de Granada. ReiDoCrea: Revista electrónica de investigación Docencia Creativa, 1, 101-105

Auné, S., Abal, F. J. P., \& Attorresi, H. F. (2016). Diseño y construcción de una escala de conducta prosocial para adultos. Revista Iberoamericana de Diagnóstico y Evaluación - e Avaliação Psicológica, 42(2), 15-25. http://doi.org/10.21865/RIDEP42_15

Auné, S., Facundo, A., \& Attorresi, H. (2017). Propiedades Psicométricas de una Prueba de Conducta Empática. Revista Iberoamericana de Diagnóstico y Evaluación - e Avaliação Psicológica. 45, 3, 47-56. https://doi.org/10.21865/RIDEP45.3.04

Bilgic, A., Bilgiç, Ö., Hergüner, S., \& Altınyazar, H.C. (2015). Autistic trait, empathy, and attention-deficit/ hyperactivity symptoms in women with idiopathic hirsutism. International Journal of Trichology, 7(3), 113-118. http://doi.org/10.4103/0974-7753.167458

Braun, S., Rosseel, Y., Kempenaers, C., Loas, G., \& Linkowski, P. (2015). Selfreport of empathy: a shortened French adaptation of the Interpersonal Reactivity Index (IRI) using two large Belgian samples. Psychological Reports: Measures \& Statistics, 117(3), 735-753. http://doi.org/10.2466/08.02.PR0.117c23z6

Brett, W., Brown, T., \& Onsman, A. (2010). Exploratory factor analysis: A five-step guide for novices. Australasian Journal of Paramedicine, 3(8), 1-13. Recuperado de http://ro.ecu.edu.au/jephc/vol8/iss3/1

Browne, M.W., \& Cudeck, R. (1993). Alternative ways of assessing model fit. Sage focus editions, 154, 136-136.

Carrasco, M.A., Delgado, B., Barbero, M.I., Holgado, F.P., \& del Barrio, M.B. (2011). Propiedades psicométricas del Interpersonal Reactivity Index (IRI) en población infantil y adolescente española. Psicothema, 23(4), 824-831.

Cheung, G. W., \& Rensvold, R. B. (2002). Evaluating goodness-of-fit indexes for testing measurement invariance. Structural Equation Modeling, 9, 233-255. https://doi.org/10.1207/S15328007SEM0902 5

Chiang, Shih-Kuang., Hua, Mau-Sun., Tam, Wai-Cheong Carl., Chao, Jian-Kang., Shiah, Yung-Jong. (2014). Developing an alternative Chinese version of the Interpersonal Reactivity Index for normal population and patients with schizophrenia in Taiwan. Brain Impairment, 15(2), 120-131. http://doi.org/10.1017/BrImp.2014.15

Chrysikou, E., \& Thompson, W. (2016). Assessing cognitive and affective empathy through the Interpersonal Reactivity Index: An argument against a two-factor 
model. Assessment, 23(6), 769- 777. http://doi.org/10.1177/1073191115599055

Cliffordson, C. (2002). The hierarchical structure of empathy: Dimensional organization and relations to social functioning. Scandinavian Journal of Psychology, 43, 49-59. http://doi.org/10.1111/1467-9450.00268

Cox, C.L., Uddin, L.Q., Di Martino, A., Castellanos, F.X., Milham, M.P., \& Kelly, C. (2012). The balance between feeling and knowing: Affective and cognitive empathy are reflected in the brain's intrinsic functional dynamics. Social Cognitive and Affective Neuroscience, 7, 727-737. http://doi.org/10.1093/scan/nsr051

Davis, M.H. (1980). A multidimensional approach to individual differences in empathy. JSAS Catalog of Selected Documents in Psychology, 10, 85

Davis, M.H. (1983). Measuring individual differences in empathy: Evidence for a multidimensional approach. Journal of Personality and Social Psychology, 44(1), 113-126. http://dx.doi.org/10.1037/0022-3514.44.1.113

Díaz, B., Rodríguez, K., \& Santa Cruz, H. (2013). Propiedades del Índice de Reactividad Interpersonal (IRI) en alumnos del ISTP "San Luis" de Trujillo. Revista de Investigación de estudiantes de Psicología "JANG”, 2(1), 143 155.

Duan, C., \& Hill, C.E. (1996). The current state of empathy research. Journal of Counseling Psychology, 43(3), 261-274. http://doi.org/10.1037/0022$\underline{0167.43 .3 .261}$

Eisenberg, N., Miller, P.A., Shell, R., Mcnalley, S., \& Shea, C. (1991). Prosocial development in adolescence: A longitudinal study. Developmental Psychology, 27, 849-857. http://dx.doi.org/10.1037/0012-1649.27.5.849

Escobar, J., \& Cuervo, A. (2008). Validez de contenido y juicio de expertos: una aproximación a su utilización. Avances en Medición, 6, 27-36.

Fernández, A. M., Dufey, M., \& Kramp, U. (2011). Testing the psychometric properties of the Interpersonal Reactivity Index (IRI) in Chile. European Journal of Psychological Assessment, 27, 179-185. http://doi.org/10.1027/1015-5759/a000065

Fernández-Pinto, I., López-Pérez, B., \& Márquez, M. (2008). Empatía: Medidas, teorías y aplicaciones en revisión. Anales de Psicología, 24(2), 284-298.

Hogan, R. (1969). Development of an Empathy Scale. Counsulting and Clinical Psychology, 33, 307-316. http://dx.doi.org/10.1037/h0027580

Horn, J. (1965). A rationale and test for the number of factors in factor analysis. Psychometrika, 30, 179-185. http://dx.doi.org/10.1007/BF02289447

Hu, L., \& Bentler, P.M. (1999). Cut-off criteria for fit indices in covariance structure analysis: conventional criteria versus new alternatives. Structural Equation Modeling, 6, 1-55. https://doi.org/10.1080/10705519909540118

Ingoglia S., Lo Coco A., \& Albiero P. (2016). Development of a brief form of the Interpersonal Reactivity Index (B-IRI). Journal of Personality Assessment, 98(5), 461-471. http://doi.org/10.1080/00223891.2016.1149858

Jöreskog, Karl., \& Sörbom, D. (2009). LISREL 8.8: User's Reference Guide, Scientific Software International, SSI. Lincolnwood: USA.

Koller, S.H., Camino, C., \& Ribeiro, J. (2001). Adaptação e validação interna de duas escalas de empatia para uso no Brasil. Estudos de Psicologia, 18(3), 4353. http://dx.doi.org/10.1590/S0103-166X2001000300004 
Kostić, J.S., Nešić, M., Stanković, M., Žikić, O., \& Marković, J. (2016). Evaluating empathy in adolescents with conduct disorders. Vojnosanit Pregled, 73(5), 429-434. http://doi.org/10.2298/VSP150121031K

Ley 10906 de Septiembre de 2006. (2006). Por la cual se reglamenta el ejercicio de la profesión de psicología, se dicta el código deontológico y bioético y otras disposiciones. Bogotá: Ministerio de Educación y Ministerio de Protección Social.

Limpo, T., Alves, R.A., \& Catro, S.L. (2010). Medir a empatia: Adaptação portuguesa do índice de reactividade interpessoal. Laboratório de Psicologia, 8(2), 171-184.

Lorenzo-Seva, U., \& Ferrando, P.J. (2015). FACTOR 10.3.01. A comprehensive program for fitting exploratory and semiconfirmatory factor analysis and IRT models. Applied Psychological Measurement, 37(6), 497-498. http://doi.org/10.1177/0146621613487794

Martin, F., Flasbeck, V., Brown, E.C., \& Brüne, M. (2017). Altered mu-rhythm suppression in borderline personality disorder. Brain Research, 1659, 64-70. http://doi.org/10.1016/j.brainres.2017.01.023

Mehrabian, A., \& Epstein, N. (1972). A measure of emotional empathy. Journal of Personality, 40, 525-543. http://doi.org/10.1111/j.1467-6494.1972.tb00078.x

Mehrabian, A. (1997). Analysis of affiliation-related traits in terms of the PAD Temperament Model. Journal of Psychology, 131,101-117. http://doi.org/10.1080/00223989709603508

Mestre, V., Frías, M. D., \& Samper, P. (2004). La medida de la empatía: análisis del Interpersonal Reactivity Index. Psicothema, 16(2), 255-260.

Mestre, V., Samper, P., \& Frias, D. (2002). Procesos cognitivos y emocionales predictores de la conducta prosocial y agresiva: la empatía como factor modulador. Psicothema, 14, 227-232.

Mestre, V., Samper, P., Tur, A., \& Díez, I. (2001). Estilos de crianza y desarrollo prosocial de los hijos. Revista de Psicología General y Aplicada, 54, 691703.

Mohamed Azahar, F.A., Mohd Fakri, N.M.R., \& Mat Pa, M.N. (2014). Associations between gender, year of study and empathy level with attitudes towards animal welfare among undergraduate doctor of veterinary medicine students in Universiti Putra Malaysia. Education in Medicine Journal, 6(4), 66-73. http://doi.org/10.5959/eimj.v6i4.320

Molina, M., Arikawa, H., \& Templer, D.I. (2013). Approval versus disapproval of dog-fighting and cockfighting among college students. Social Behavior \& Personality: An International Journal, 41(2), 345-352.

Montag, C., Brockmann, E.M., Lehmann, A, Müller, D.J., Rujescu, D., \& Gallinat, J. (2012). Association between oxytocin receptor gene polymorphisms and selfrated 'empathic concern' in Schizophrenia. PLoS ONE 7(12), e51882. http://doi.org/10.1371/journal.pone.0051882

Montero, I., \& León, O.G. (2007). A guide for naming research studies in Psychology. International Journal of clinical and Health Psychology, 7(3), 847-862.

Pérez-Albéniz, A., de Paul, J., Etxeberría, J., Paz, M., \& Torres, E. (2003). Adaptación de Interpersonal Reactivity Index (IRI) al español. Psicothema,15(2), 267-272. 
Pulos, S., Elison, J., \& Lennon, R. (2004). The hierarchical structure of the Interpersonal Reactivity Index. Social Behavior and Personality, 32, 355-360. https://doi.org/10.2224/sbp.2004.32.4.355

Resolución 8430 de 1993. (1993). Normas científicas, técnicas y administrativas para la investigación en salud. Ministerio de Salud. Colombia.

Sampaio, L.R., Guimarães, P.R.B., Camino, C.P.S., Formiga, N.S., \& Menezes, I.G. (2011). Estudos sobre a dimensionalidade da empatia: Tradução e adaptação do Interpersonal Reactivity Index (IRI). Psico, 42(1), 67-76.

Samper, P., Díez, E., \& Martí, M. (1998). Razonamiento moral y empatía. I jornada de psicología del pensamiento. Universidade de santiago de Compostela, 389-404.

Solano-Flores, G., Contreras-Niño, L.A., \& Backhoff, E. (2006). Traducción y adaptación de pruebas: Lecciones aprendidas y recomendaciones para países participantes en TIMSS, PISA y otras comparaciones internacionales. Revista Electrónica de Investigación Educativa (REDIE), 8(2), 2-21.

Song, Y., \& Shi, M. (2017). Associations between empathy and big five personality traits among Chinese undergraduate medical students. PLOS ONE 12(2), e0171665. http://doi.org/10.1371/journal. pone.0171665

Statistic Package for Social Sciences (2013). IBM-SPSS for Windows, version 22.0. Chicago: SPSS Inc.

Stratton, T.D., Saunders, J.A., \& Elam, C.L. (2008). Changes in medical students' emotional intelligence: An exploratory study. Teaching and Learning in Medicine, 20(3), 279-284. http://doi.org/10.1080/10401330802199625

Tamura, A., Sugiura, Y., Sugiura, T., \& Moriya, J. (2016). Attention moderates the relationship between primary psychopathy and affective empathy in undergraduate students. Psychological Reports, 119(3), 608-629. http://doi.org/10.1177/0033294116667699

Wang, Q., Zhang, Z., Dong, F., Chen, L., Zheng, L., Guo, X., \& Li, J. (2014). Anterior insula GABA levels correlate with emotional aspects of empathy: A proton magnetic resonance spectroscopy study. PLoS ONE, 9(11), e113845. http://doi.org/10.1371/journal.pone.0113845 


\section{Chaparro ClaviJo, \& Pineda-Roa}

\section{Anexo A}

Inventario de reactividad interpersonal IRI

Preocupación empática

2. A menudo tengo sentimientos de compasión y preocupación hacia la gente menos afortunada que yo.

4. Algunas veces no me siento muy triste por otras personas cuando tienen problemas.

9. Cuando veo que se están aprovechando de alguien tiendo a protegerlo/a.

14. Normalmente las desgracias de otras personas no me molestan mucho.

18. A veces no siento mucha compasión cuando veo que alguien está siendo tratado/a injustamente.

20. A menudo estoy bastante conmovido/a por cosas que veo que ocurren.

22. Me describiría como una persona con un corazón muy blando.

\section{Fantasía}

5. Verdaderamente me identifico con los sentimientos de los personajes de una novela.

7. Usualmente soy objetivo/a cuando veo una película u obra de teatro y no me involucro completamente en ella.

16. Después de ver una obra de teatro o una película me he sentido como si fuera uno de los personajes.

23. Cuando veo una buena película puedo situarme muy fácilmente en el lugar del protagonista.

26. Cuando estoy leyendo una historia o una novela interesante, imagino cómo me sentiría si los acontecimientos de la historia me estuvieran sucediendo a mí.

\section{Toma de perspectiva}

8. Antes de tomar una decisión intento tener en cuenta el punto de vista de todos los que están en desacuerdo.

11. A veces intento comprender mejor a mis amigos/as imaginándome cómo ven ellos/as las cosas desde su punto de vista.

21. Creo que hay dos partes para cada cuestión e intento tener en cuenta ambas partes.

25. Cuando estoy disgustado/a con alguien, normalmente intento ponerme en su lugar por un momento.

28. Antes de criticar a alguien intento imaginar cómo me sentiría si estuviera en su lugar.

$$
\text { Aflicción personal }
$$

6. En situaciones de emergencia me siento aprensivo/a e incómodo/a.

10. Algunas veces me siento impotente cuando estoy en la mitad de una situación muy emocional.

17. Me asusta estar en una situación emocionalmente tensa.

24. Tiendo a perder el control durante las emergencias.

27. Me derrumbo cuando veo a alguien que necesita desesperadamente ayuda en una emergencia.

1. Con cierta regularidad sueño y fantaseo acerca de las cosas que me podrían suceder*.

3. A veces encuentro difícil ver las cosas desde el punto de vista de otra persona*.

12. Es raro para mí involucrarme completamente en un buen libro o película*.

13. Cuando veo que lastiman a alguien tiendo a permanecer calmado/a*.

15. Si estoy seguro/a que tengo la razón en algo no pierdo mucho tiempo escuchando los argumentos de los demás*.

19. Normalmente soy bastante efectivo/a para manejar emergencias*.

*Ítem excluidos con correlación ítem total por debajo de .30 